\title{
The underlying molecular mechanism and potential drugs for treatment in papillary renal cell carcinoma: A study based on TCGA and Cmap datasets
}

\author{
JIN-SHU PANG ${ }^{1}$, ZHE-KUN LI $^{1}$, PENG LIN $^{2}$, XIAO-DONG WANG ${ }^{2}$, GANG CHEN $^{1}$, \\ HAI-BIAO YAN ${ }^{3}$ and SHENG-HUA LI ${ }^{3}$
}

Departments of ${ }^{1}$ Pathology, ${ }^{2}$ Medical Ultrasonics and ${ }^{3}$ Urology, The First Affiliated Hospital of Guangxi Medical University, Nanning, Guangxi 530021, P.R. China

Received August 9, 2018; Accepted February 5, 2019

DOI: 10.3892/or.2019.7014

\begin{abstract}
Papillary renal cell carcinoma (PRCC) accounts for $15-20 \%$ of all kidney neoplasms and continually attracts attention due to the increase in the incidents in which it occurs. The molecular mechanism of PRCC remains unclear and the efficacy of drugs that treat PRCC lacks sufficient evidence in clinical trials. Therefore, it is necessary to investigate the underlying mechanism in the development of PRCC and identify additional potential anti-PRCC drugs for its treatment. The differently expressed genes (DEGs) of PRCC were identified, followed by Gene Ontology and Kyoto Encyclopedia of Genes and Genomes (KEGG) enrichment analyses for functional annotation. Then, potential drugs for PRCC treatment were predicted by Connectivity Map (Cmap) based on DEGs. Furthermore, the latent function of query drugs in PRCC was explored by integrating drug-target, drug-pathway and drug-protein interactions. In total, 627 genes were screened as DEGs, and these DEGs were annotated using KEGG pathway analyses and were clearly associated with the complement and coagulation cascades, amongst others. Then, 60 candidate drugs, as predicted based on DEGs, were obtained from the Cmap database. Vorinostat was considered as the most promising drug for detailed discussion. Following protein-protein interaction (PPI) analysis and molecular docking, vorinostat
\end{abstract}

Correspondence to: Professor Sheng-Hua Li, Department of Urology, The First Affiliated Hospital of Guangxi Medical University, No. 6 Shuangyong Road, Nanning, Guangxi 530021, P.R. China E-mail: 13877115066@163.com

Abbreviations: PRCC, papillary renal cell carcinoma; TCGA, The Cancer Genome Atlas; DEGs, differently expressed genes; GO, Gene Ontology; KEGG, Kyoto Encyclopedia of Genes and Genomes; Cmap, Connectivity Map; CCRCC, clear renal cell carcinoma; CAMs, cell adhesion molecules

Key words: papillary renal cell carcinoma, differently expressed genes, underlying mechanisms, molecular docking and potential drugs was observed to interact with $\mathrm{C} 3$ and ANXN1 proteins, which are the upregulated hub genes and may serve as oncologic therapeutic targets in PRCC. Among the top 20 metabolic pathways, several significant pathways, such as complement and coagulation cascades and cell adhesion molecules, may greatly contribute to the development and progression of PRCC. Following the performance of the PPI network and molecular docking tests, vorinostat exhibited a considerable and promising application in PRCC treatment by targeting $\mathrm{C} 3$ and ANXN1.

\section{Introduction}

Papillary renal cell carcinoma (PRCC) is the second most prevalent subtype of renal cell carcinoma (RCC) and it represents $15-20 \%$ of all kidney neoplasms (1-4). Compared with patients with clear RCC (CCRCC), those with PRCC generally have a more favorable outcome following surgical treatment and these patients are less likely to exhibit distant metastasis and recurrence (5-7). However, as incidents of RCC continue to increase, representing 2-3\% of all adult malignancies, PRCC still receives attention as the second most common subtype of RCC (8-10). Although many genes have been demonstrated to be involved in the development of PRCC in recent years (11), the underlying molecular mechanism of PRCC still remains uncertain. Regarding the PRCC treatment, several agents, such as mechanistic target of rapamycin suppressor and anti-vascular endothelial growth factor agents, are suitable options for patients with progressive and metastatic PRCC, following the support of clinical trials consisting of all RCC subtypes (12). However, specific evidence from patients affected by PRCC is insufficient and controversial, and results from the limited amount of small samples (12). Therefore, it is necessary to investigate the underlying mechanisms of PRCC and search for additional ignored drugs for its treatment.

Although it is difficult to support expenditure in researching novel oncologic therapeutic drugs, the clinical application of a newly-discovered drug typically requires long-term trials to ensure its safety and tolerance in the human body (13). Therefore, the repurposing of known drugs is a feasible drug development strategy, which provides substantial advantages 
in meeting the high demands of better therapeutic agents in anti-PRCC treatment by searching for more suppressors with known safety but ignored oncologic chemotherapy.

In the present study, the genes that are expressed differently in cases of patients with PRCC and non-PRCC controls were screened using The Cancer Genome Atlas (TCGA) data. A bioinformatics analysis, including Gene Ontology (GO) analysis and Kyoto Encyclopedia of Genes (KEGG) enrichment pathway, was performed to explore the underlying mechanism in PRCC. Next, the Connectivity map (Cmap), which is a database that contains $>6,900$ expression profiles and 1,309 compounds, was used to search for potential drugs for PRCC treatment based on differently expressed genes (DEGs). Then, the hub genes of DEGs in PRCC were selected following the construction of protein-protein interaction (PPI) network analysis, and molecular docking tests between query drugs and hub genes were further performed to validate the prospective application in PRCC treatment.

\section{Materials and methods}

Identification of differently expressed genes based on TCGA data. The TCGA database, which comprises 33 cancer types and $>10,000$ samples, has been widely used to investigate the underlying mechanism in human cancers. GEPIA (14), which is an online tool based on the TCGA data, can be used for DEGs, correlation, survival, and co-expressed genes analyses in various types of cancer. In the present study, GEPIA was used to analyze the DEGs between PRCC tissues and non-PRCC adjacent tissues with one-way analysis of variance (ANOVA) method and Tukey's test, and genes with a q-value $<0.05$ and $\mid \log 2$ fold-change (FC) $\mid>2$ were secerned and considered as the significant DEGs.

Functional annotation and KEGG pathway enrichment analysis. For the investigation of the underlying mechanism of PRCC, gene functional annotation and KEGG pathways analyses were performed to explore how aforementioned DEGs function in the onset and development of PRCC (15-17). MetaScape (18) (http://metascape.org), which was updated in 2018 , is a web-based tool that provides gene functional annotation and enrichment analysis. In the present study, GO analysis was performed for the gene function annotation by MetaScape, and another tool, Webgestalt (ORA method) (19) (http://www.webgestalt.org), was applied for KEGG pathway enrichment analysis to illustrate which pathways may contribute to the occurrence of PRCC.

Prediction of potential drugs for PRCC treatment based on DEGs by Cmap and Drug Pair Seeker. The Cmap database $(20,21)$ uses gene-expression signatures to predict small molecular compounds for a specific disease. In the present study, the DEGs of PRCC were divided into 2 groups: Upregulated and downregulated genes. Upregulated and downregulated genes were subsequently uploaded to the Cmap in the 'query' page, and searches for small molecule drugs that may treat PRCC were performed. Scores ranging from -1-1 represented the correlation between the drug and DEGs. The more negatively correlated drugs indicate greater correlation with the uploaded DEGs and are more likely to be used for PRCC treatment. In the present study, drugs with a score of $\leq 0.75$ were considered as candidate drugs for PRCC treatment. Additionally, Drug Pair Seeker (DPS, version 1.4.0, http://www.maayanlab.net/DPS/) was also utilized to predict which drug from Old AFFY Cmap data could be correlated with the query drug together to reverse the direction of gene expression (22).

Construction of the drug-pathway network. For the exploration of the associations between candidate agents and pathways, the expression profiles for each of the candidate agents were downloaded and the genes affected by candidate drugs were obtained from the Cmap database. The Cmap incorporates 6,100 instances for 1,309 small molecular agents, and each instance includes gene expression profiles of control and corresponding treatment for a certain agent. For each instance, expression profiles of treatment and control were matched for the previously listed candidate drugs according to descriptions of the annotation file. Then, DEGs were identified between control and treatment with $\log 2 \mathrm{FC}>1$ or $\leq 1$ (ORA method) for each candidate drug, and these DEGs were considered as genes that were affected by certain drugs. Finally, these affected genes for each above candidate molecules were entered into SubpathwayMiner $(3,23)$ (an R package for identifying subpathways depend on the KEGG database) to identify significantly enriched subpathways, and a subpathway with false discovery rate (FDR) $<0.1$ was considered as statistically significant.

Construction of drug-target networks. To further explore the potential mechanism of the top 10 prospective drugs, the SMILE structure of these drugs was obtained from the DrugBank database (https://www.drugbank.ca/). Next, the STITCH database (24) was applied to identify targets of these drugs, and then drug-target networks were construed to show the interactions between the top 10 drugs and their corresponding targets.

Further exploration of query drugs for PRCC treatment. Many types of dysregulated genes are involved in tumorigenesis. To elucidate which genes may serve as key roles in such a complex connection network, a PPI network was constructed for the DEGs of PRCC using the STRING database (25), followed by the identification of the hub genes by CentiScape (26), a plugin of Cytoscape, which may have a key role in the gene regulation network. Next, the expression levels of hub genes were determined by TCGA data in GEPIA. Finally, immunohistochemical (IHC) results of complement C3 (C3) and annexin 1 (ANXA1) in various types of cancers were acquired from the Human Protein Atlas (27) (version 18, https://www.proteinatlas.org/; ANXA1, https://www. proteinatlas.org/ENSG00000135046-ANXA1/pathology; C3, https://wwwhttps://www.proteinatlas.org/ENSG00000125730-C3/ pathology), from which the prognostic value of C3 and ANXA1 based on TCGA data (ANXA1, https://www.proteinatlas.org/ ENSG00000135046-ANXA1/pathology/tissue/renal+cancer/ KIRP; C3, https://www.proteinatlas.org/ENSG00000125730$\mathrm{C} 3 /$ pathology/tissue/renal+cancer/KIRP), as well as IHC images in renal cancer and normal kidney were also obtained (C3 protein in normal kidney, https://www. proteinatlas.org/ENSG00000125730-C3/tissue/kidney\#; C3 
Table I. Significant GO terms for each GO category enriched by MetaScape.

\begin{tabular}{|c|c|c|c|c|}
\hline Categories & GO ID & GO terms & Gene numbers & $\log _{10}(\mathrm{P}$-value $)$ \\
\hline \multirow[t]{10}{*}{ Biological processes } & GO:0006820 & Anion transport & 58 & -17.90430 \\
\hline & GO:0050801 & Ion homeostasis & 65 & -17.56780 \\
\hline & GO:0001822 & Kidney development & 35 & -15.00420 \\
\hline & GO:0072358 & Cardiovascular system development & 59 & -14.05610 \\
\hline & GO:0007588 & Excretion & 18 & -13.69620 \\
\hline & GO:0055067 & Monovalent inorganic cation homeostasis & 23 & -11.67040 \\
\hline & GO:0050878 & Regulation of body fluid levels & 43 & -11.62370 \\
\hline & GO:0007169 & $\begin{array}{l}\text { Transmembrane receptor protein tyrosine kinase } \\
\text { signaling pathway }\end{array}$ & 50 & -10.33970 \\
\hline & GO:0048871 & Multicellular organismal homeostasis & 33 & -10.24460 \\
\hline & GO:0043062 & Extracellular structure organization & 35 & -9.96938 \\
\hline \multirow[t]{10}{*}{ Cellular components } & GO:0045177 & Apical part of cell & 55 & -26.49700 \\
\hline & GO:0016323 & Basolateral plasma membrane & 36 & -19.19540 \\
\hline & GO:0031012 & Extracellular matrix & 46 & -11.64710 \\
\hline & GO:0009986 & Cell surface & 50 & -9.54270 \\
\hline & GO:0031225 & Anchored component of membrane & 17 & -6.36750 \\
\hline & GO:0005911 & Cell-cell junction & 28 & -5.48420 \\
\hline & GO:0072562 & Blood microparticle & 17 & -5.43264 \\
\hline & GO:0031526 & Brush border membrane & 9 & -5.35366 \\
\hline & GO:0005902 & Microvillus & 11 & -5.14557 \\
\hline & GO:0000323 & Lytic vacuole & 37 & -2.91416 \\
\hline \multirow[t]{10}{*}{ Molecular functions } & GO:0005539 & Glycosaminoglycan binding & 27 & -11.27660 \\
\hline & GO:0008509 & Anion transmembrane transporter activity & 34 & -10.93000 \\
\hline & GO:0019199 & Transmembrane receptor protein kinase activity & 15 & -8.91288 \\
\hline & GO:0015081 & Sodium ion transmembrane transporter activity & 20 & -8.45459 \\
\hline & GO:0004857 & Enzyme inhibitor activity & 30 & -7.03172 \\
\hline & GO:0005509 & Calcium ion binding & 40 & -5.9180 \\
\hline & GO:0004252 & Serine-type endopeptidase activity & 21 & -5.86497 \\
\hline & GO:0019825 & Oxygen binding & 9 & -5.83118 \\
\hline & GO:0033293 & Monocarboxylic acid binding & 10 & -5.32175 \\
\hline & GO:0019838 & Growth factor binding & 14 & -5.29116 \\
\hline
\end{tabular}

GO, Gene Ontology.

protein in renal cancer, https://www.proteinatlas. org/ENSG00000125730-C3/pathology/tissue/renal+cancer\#; ANXA1 protein in normal kidney, https://www.proteinatlas. org/ENSG00000135046-ANXA1/tissue/kidney\#; ANXA1 protein in renal cancer, https://www.proteinatlas.org/ ENSG00000135046-ANXA1/pathology/tissue/renal+cancer\#).

To further investigate the potential application of query drugs in PRCC treatment, molecular docking study, as determined by systemsDock (28), a web-online tool for network pharmacology-based prediction and analyses, was performed to simulate the drug-protein interactions between the query drugs and hub genes. This tool provides a high-precision docking simulation and docking pattern map to systematically illustrate the ligand selectivity and the interaction ability between a ligand and proteins, as well as to elucidate how a specific ligand acts on a complex protein. The interaction ability between the query drug and proteins are assessed by docking scores. A drug that interacts well with ANXA1 (PDB code: 1HM6) and C3 (PDB code: 1GHQ; docking score $>4$ ) may have a better anticancer application prospect in PRCC as it suppresses the gene regulation network by inhibiting hub genes. A flow chart detailing the experimental design of the present study is presented in Fig. S1.

Statistical analysis. To analyze the genes that are differently expressed in PRCC and non-PRCC adjacent tissues, the one-way ANOVA method and Tukey's test was applied and genes with a q-value $<0.05$ and $\log 2 \mathrm{FCl}>2$ were selected as the significant DEGs. For GO and KEGG pathway analysis, the overrepresentation enrichment analysis method was used, in which a GO term or pathway with $\mathrm{P}<0.05$ was significant. Following the determination of hub genes among the above DEGs, Kaplan-Meier survival curves were performed to explore their prognostic value in patients with PRCC. Furthermore, the 
Table II. Significant KEGG pathways enriched by Webgestalt.

\begin{tabular}{llrr}
\hline Pathway ID & \multicolumn{1}{c}{ KEGG pathway } & Counts & P-value \\
\hline hsa04610 & Complement and coagulation cascades & 15 & $<0.00001$ \\
hsa04514 & Cell adhesion molecules & 18 & $<0.00010$ \\
hsa04978 & Mineral absorption & 10 & $<0.00001$ \\
hsa04966 & Collecting duct acid secretion & 7 & $<0.00001$ \\
hsa04960 & Aldosterone-regulated sodium reabsorption & 8 & 0.00010 \\
hsa04670 & Leukocyte transendothelial migration & 14 & 0.00018 \\
hsa05323 & Rheumatoid arthritis & 10 & 0.00250 \\
hsa04270 & Vascular smooth muscle contraction & 12 & 0.00255 \\
hsa00590 & Arachidonic acid metabolism & 8 & 0.00261 \\
hsa05110 & Vibrio cholerae infection & 7 & 0.00336 \\
hsa00260 & Glycine, serine and threonine metabolism & 6 & 0.00417 \\
hsa00010 & Glycolysis/Gluconeogenesis & 8 & 0.00426 \\
hsa04020 & Calcium signaling pathway & 15 & 0.00480 \\
hsa04976 & Bile secretion & 8 & 0.00608 \\
hsa00980 & Metabolism of xenobiotics by cytochrome P450 & 8 & 0.00779 \\
hsa04015 & Rap1 signaling pathway & 16 & 0.00842 \\
hsa04971 & Gastric acid secretion & 8 & 0.00843 \\
hsa04961 & Endocrine and other factor-regulated calcium reabsorption & 6 & 0.00929 \\
hsa00350 & Tyrosine metabolism & 5 & 0.01079 \\
hsa04614 & Renin-angiotensin system & 4 & 0.01113 \\
\hline
\end{tabular}

KEGG, Kyoto Encyclopedia of Genes and Genomes.

one-way ANOVA method and Tukey's test was also utilized to identify the genes affected by each candidate drug $(\log 2 \mathrm{FC}>1$ or $\leq 1)$. Thereafter, these affected genes were used to explore significantly enriched subpathways $($ FDR $<0.1)$ affected by candidate agents with SubpathwayMiner tool.

\section{Results}

GO analysis and KEGG pathway enrichment analysis using DEGs of PRCC. Altogether, 627 DEGs were identified from TCGA data, including 161 upregulated and 466 downregulated genes (Fig. S2). The GO analysis includes three categories (biological process, molecular function, and cellular component), and the 10 significant enrichment terms were displayed for each category (Table I). From the biological process (Fig. 1A), it was observed that DEGs were predominantly associated with anion transport, ion hemostasis and kidney development. For the cellular component (Fig. 1B), these DEGs were enriched in the apical part of the cell, extracellular matrix and basolateral plasma membrane. In molecular function (Fig. 1C), these DEGs were associated with glycosaminoglycan binding, anion transmembrane transporter activity, and calcium ion binding. Regarding the KEGG pathway (Fig. 2; Table II), the results demonstrated that DEGs are significantly associated with complement and coagulation cascades, cell adhesion molecules and mineral absorption.

The drugs of repurposing for PRCC treatment. From the prediction of the Cmap dataset, 60 candidate drugs (after removing duplicates) that scored $\leq-0.75$ were considered as potential drugs for PRCC treatment (Table SI). The information of the 10 drugs with significant scores is listed in Table III and their 2D molecular structures, as provided by Drugbank (https://www.drugbank.ca/), are displayed in Fig. S3.

Construction of drug-pathway and drug-target network for candidate drugs. In total, 8 small molecular components among 60 candidate drugs are significantly associated with 9 metabolic pathways (Table SII and Fig. 3). For the 10 significant drugs, the corresponding pathways of vorinostat are p53 and MAPK signing pathway, and the p53 signaling pathway is affected by chlorprothixene. Then, the targets were predicted for the top 10 drugs using the STITCH database; however the targets of pinacidil, ciclosporin, and metacycline were not available. Therefore, only 7 drug-target networks are presented (Fig. 4).

Molecular docking study and drug pairing prediction for vorinostat. Regarding hub genes in the regulation network of DEGs, 9 genes (BDKRB2, C3, PLG, EGF, IGF2, KNG1, CASR, ANXA1 and ADCY4) were selected as hub genes due to their centrality degree $\geq 15$ (Fig. 5). Among these 9 hub genes, 7 genes are significantly downregulated and 2 genes (C3 and ANXA1) are significantly upregulated (Fig. 6), which suggests that $\mathrm{C} 3$ and ANXA1 may serve as potential therapeutic targets in the chemotherapy of PRCC. The prognostic value of $\mathrm{C} 3$ and ANXA1, as determined by TCGA data, is presented in Fig. 7. Furthermore, the validation of protein 
A

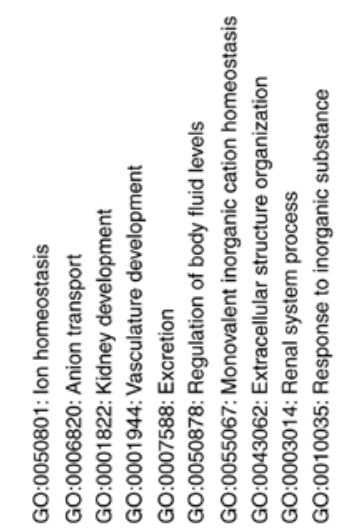

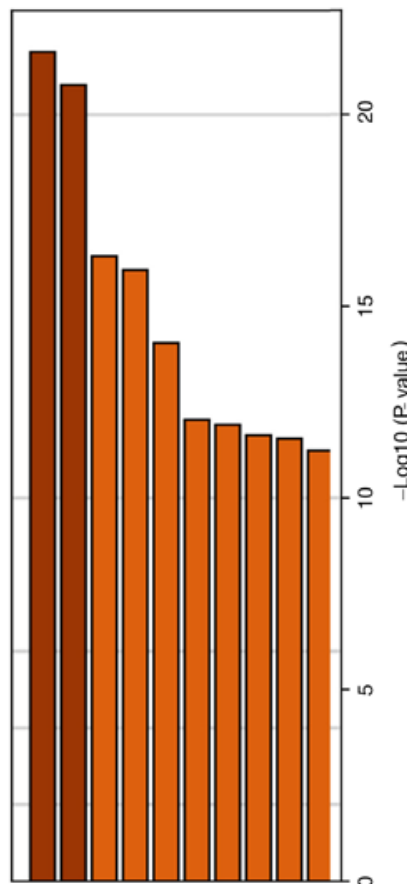

B
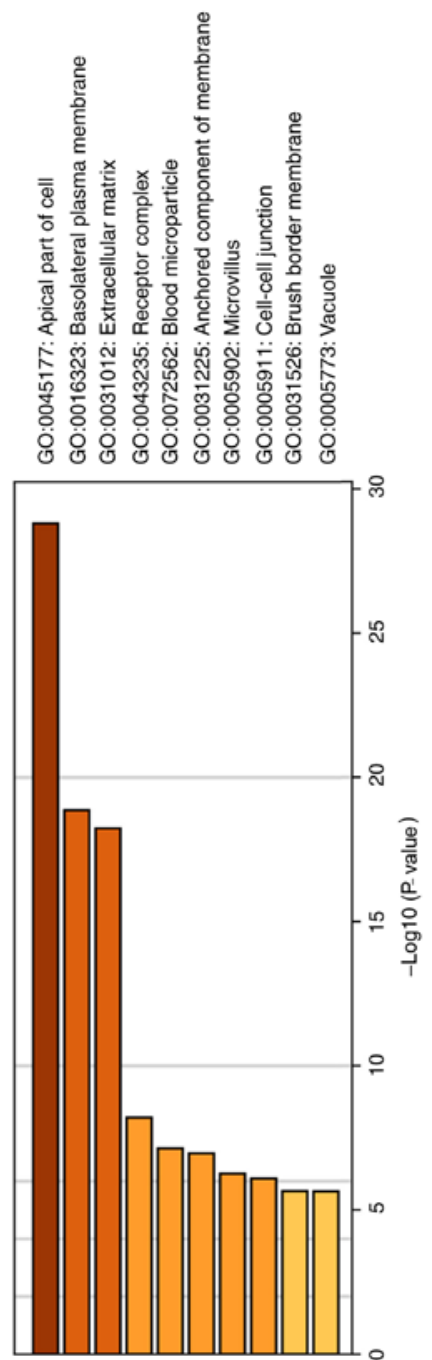

C

(3)

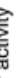

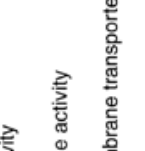

글

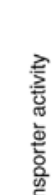

毫

롤

旅

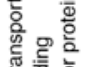

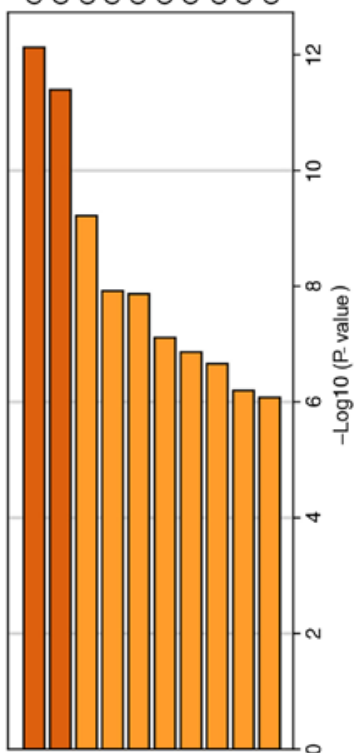

Figure 1. The GO enrichment analysis using differentially expressed genes of papillary renal cell carcinoma. (A) Biological process. (B) Cellular component. (C) Molecular function. GO, Gene Ontology. The color intensity of bars indicated the P-value of the corresponding term.

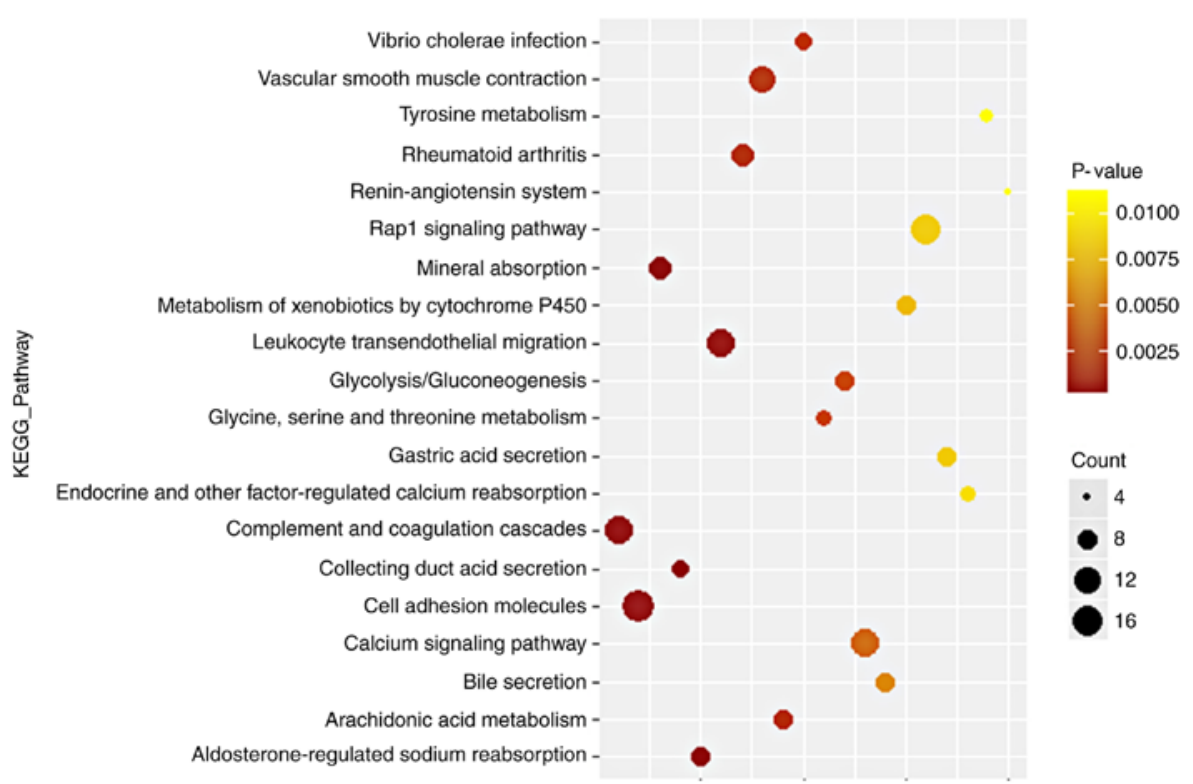

Figure 2. The KEGG pathway analysis using differentially expressed genes of papillary renal cell carcinoma. KEGG, Kyoto Encyclopedia of Genes and Genomes. 
Table III. Information of the 10 prospective drugs with significant scores for papillary renal cell carcinoma treatment.

\begin{tabular}{|c|c|c|c|}
\hline Drug name & Molecular formula & Classification & Clinical application \\
\hline Pinacidil & $\mathrm{C}_{13} \mathrm{H}_{19} \mathrm{~N}_{5}$ & Membrane transport modulators & Antihypertension \\
\hline Ciclosporin & $\mathrm{C}_{62} \mathrm{H}_{111} \mathrm{~N}_{11} \mathrm{O}_{12}$ & Immunosuppressant & $\begin{array}{l}\text { Transplant rejection, rheumatoid arthritis, and } \\
\text { severe psoriasis }\end{array}$ \\
\hline Naftifine & $\mathrm{C}_{21} \mathrm{H}_{21} \mathrm{~N}$ & Antifungal agent & Tinea pedis, tinea cruris, and tinea corporis \\
\hline Vorinostat & $\mathrm{C}_{14} \mathrm{H}_{20} \mathrm{~N}_{2} \mathrm{O}_{3}$ & Histone deacetylase suppressor & Cutaneous T-cell lymphoma \\
\hline Metacycline & $\mathrm{C}_{22} \mathrm{H}_{22} \mathrm{~N}_{2} \mathrm{O}_{8}$ & Tetracycline antibiotic agent & Acute bacterial exacerbations of chronic bronchitis \\
\hline Sulfacetamide & $\mathrm{C}_{8} \mathrm{H}_{10} \mathrm{~N}_{2} \mathrm{O}_{3} \mathrm{~S}$ & Sulfonamide antibiotic agent & $\begin{array}{l}\text { Bacterial vaginitis, keratitis, acute conjunctivitis, } \\
\text { and blepharitis }\end{array}$ \\
\hline Chlorprothixene & $\mathrm{C}_{18} \mathrm{H}_{18} \mathrm{C}_{1} \mathrm{NS}$ & Antipsychotic agent & Psychotic disorders, and acute mania \\
\hline Amiodarone & $\mathrm{C}_{25} \mathrm{H}_{29} \mathrm{I}_{2} \mathrm{NO}_{3}$ & Anti-Arrhythmia agent & $\begin{array}{l}\text { Frequently recurring ventricular fibrillation and } \\
\text { tachycardia }\end{array}$ \\
\hline Noretynodrel & $\mathrm{C}_{20} \mathrm{H}_{26} \mathrm{O}_{2}$ & Hormonal agent & Gynecological disorders, and contraceptives \\
\hline Valproic acid & $\mathrm{C}_{8} \mathrm{H}_{16} \mathrm{O}_{2}$ & Anti-epileptic Agent & Epilepsy, mania, and migraine headache \\
\hline
\end{tabular}

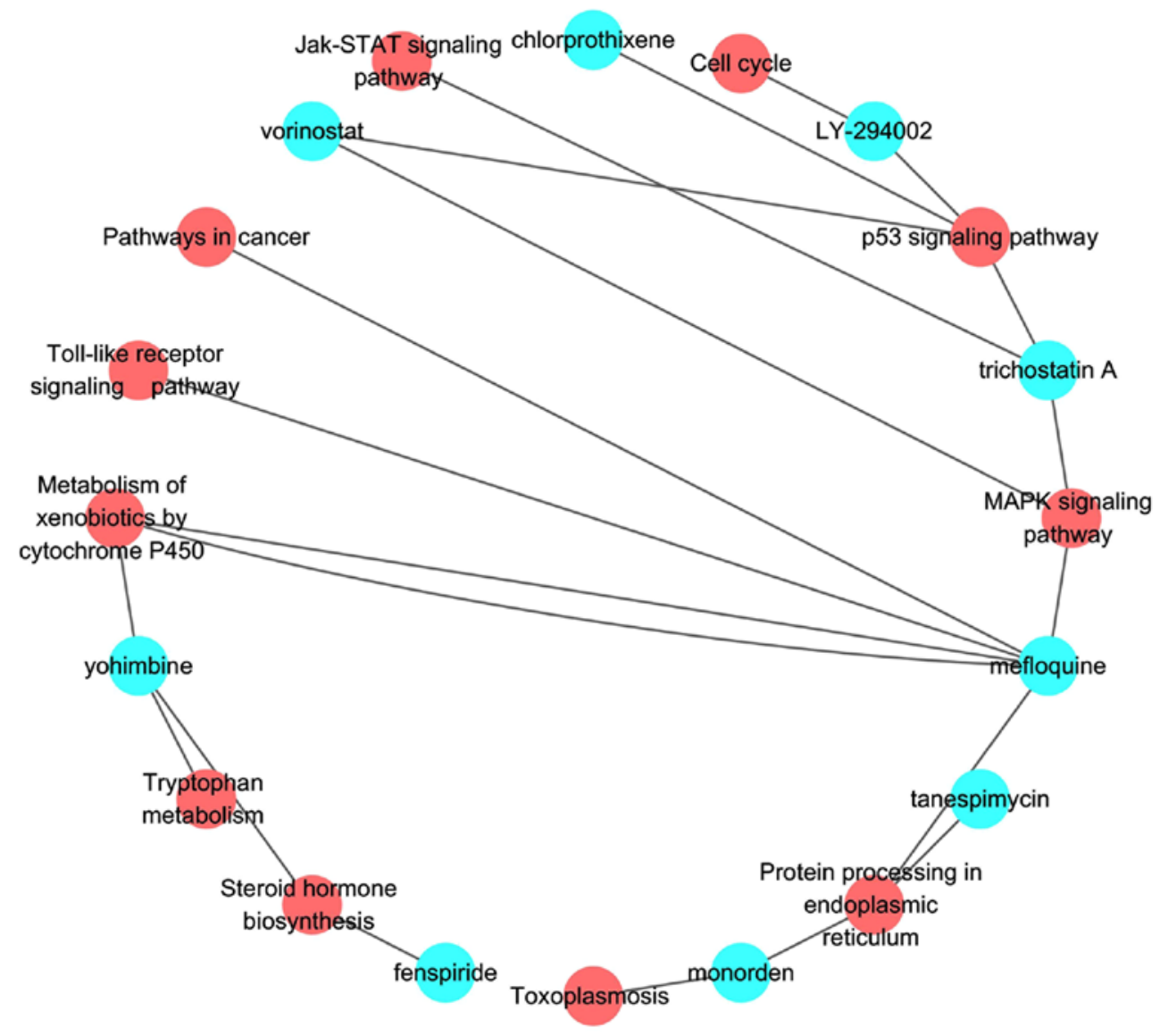

Figure 3. The drug-pathway network to indicate pathways significantly affected by potential therapy drugs of papillary renal cell carcinoma. The blue nodes represent drugs, and the red nodes represent the pathways affected by drugs.

levels for C3 and ANXA1 in various types of tumors is presented in Figs. 8 and 9. Distinctly positive C3 protein was observed in tumors' stromal and the majority of malignant cells displayed weak-to-moderate cytoplasmic immunoreactivity. Similarly, most malignant cells displayed moderate-to-strong cytoplasmic and nuclear positivity of ANXA1 protein except breast cancers and malignant lymphomas. Regarding renal cancer tissues, 7 of $13(53.8 \%)$ renal cancer tissues exhibited high/medium C3 protein expression and 9 of 12 (75\%) renal cancer tissues exhibited high/medium ANXA1 protein expression. Nevertheless, the expression difference of C3 and ANXN1 protein in renal cancer and non-cancer kidney is not well demonstrated due to the limited controls. Among the top 10 agents, vorinostat was reported to be closely 

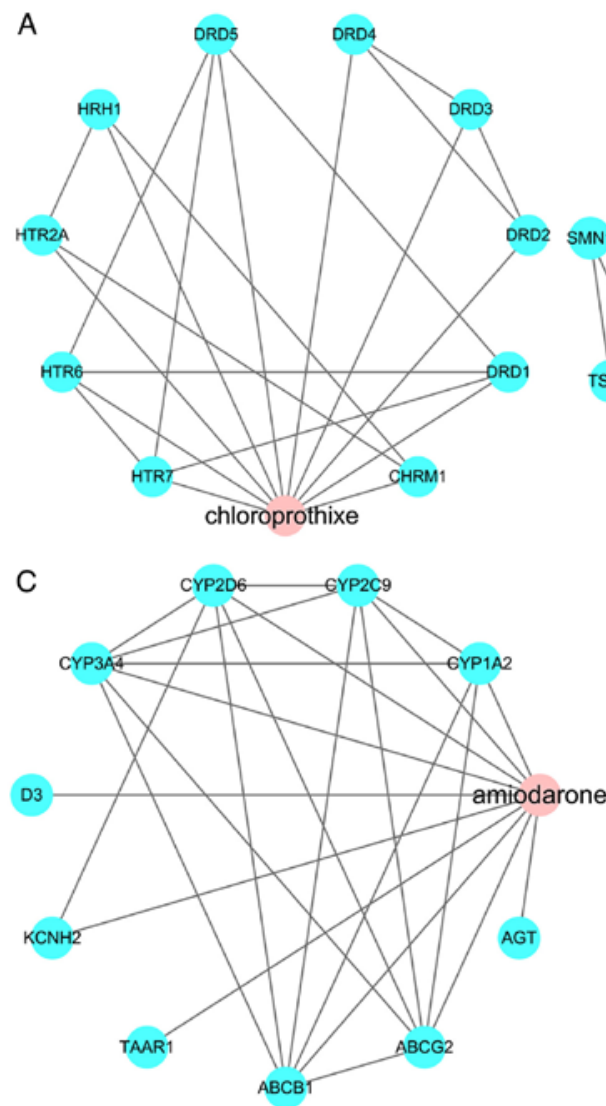

$\mathrm{E}$

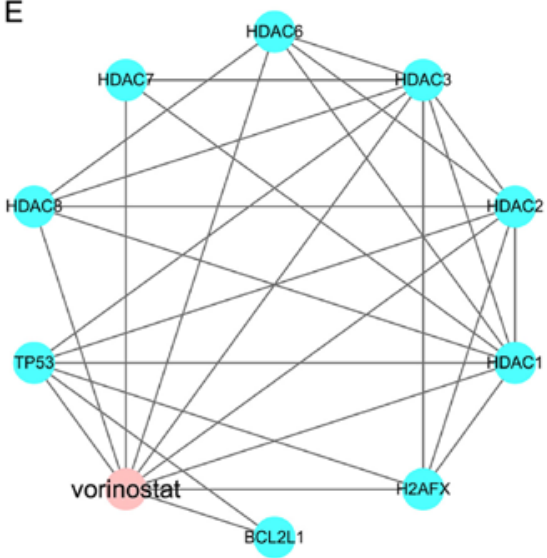

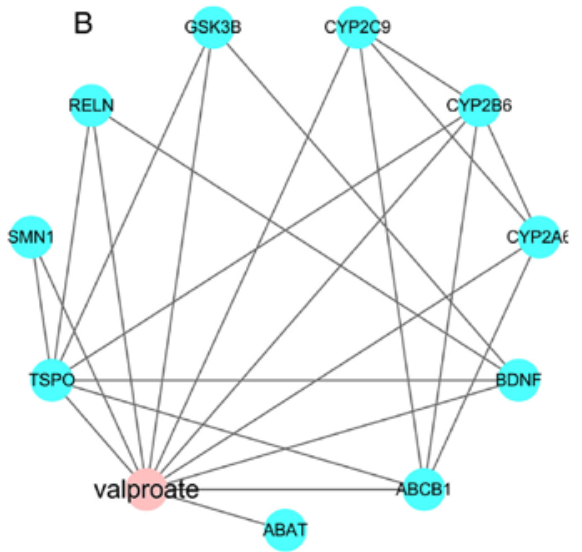

D

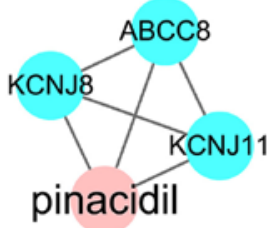

$\mathrm{F}$

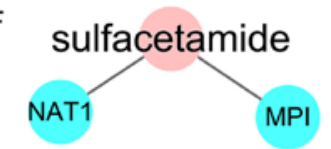

G

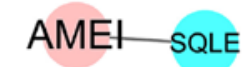

Figure 4. The drug-target networks for 7 of the 10 drugs with significant scores constructed by the STITCH database. (A) Ahlorprothixene; (B) valproate (valproic acid); (C) amiodarone; (D) pinacidil; (E) vorinostat; (F) sulfacetamide; and (G) AMEI (naftifine). The targets of pinacidil, ciclosporin, and metacycline are not available in STITCH database.

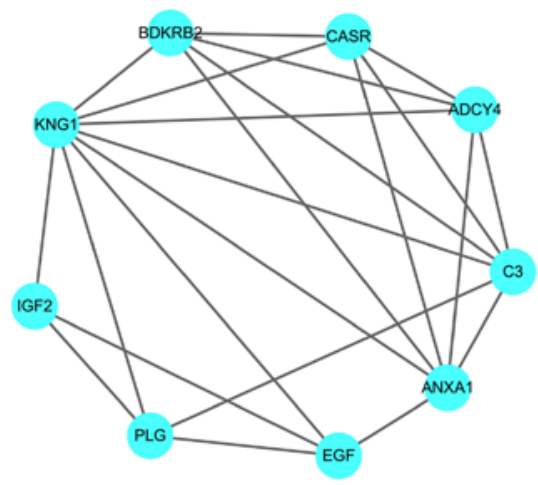

Figure 5. The protein-protein interaction of hub genes in the gene regulation network of papillary renal cell carcinoma. correlated with cell cycle and had been repurposing for the patients with progressive cutaneous T-cell lymphoma (29-32). Consequently, the potential chemotherapy effect of vorinostat in PRCC patients seems to be a feasible investigation. Notably, the molecular docking tests indicate that vorinostat can interact well with ANXA1 and $\mathrm{C} 3$ proteins, and that the docking scores for ANXA1 (PDB code: 1HM6; Fig. 10A-C) and C3 (PDB code: 1GHQ; Fig. 10D-F) are 4.866 and 4.634, respectively. Furthermore, the docking results suggest that vorinostat is a potentially prospective agent to treat and reverse PRCC by interfering with gene regulation network through targeting C3 and ANXN1. In addition, the DPS program computationally-predicted which drugs would improve the reversal effects of gene expression changes when combined 

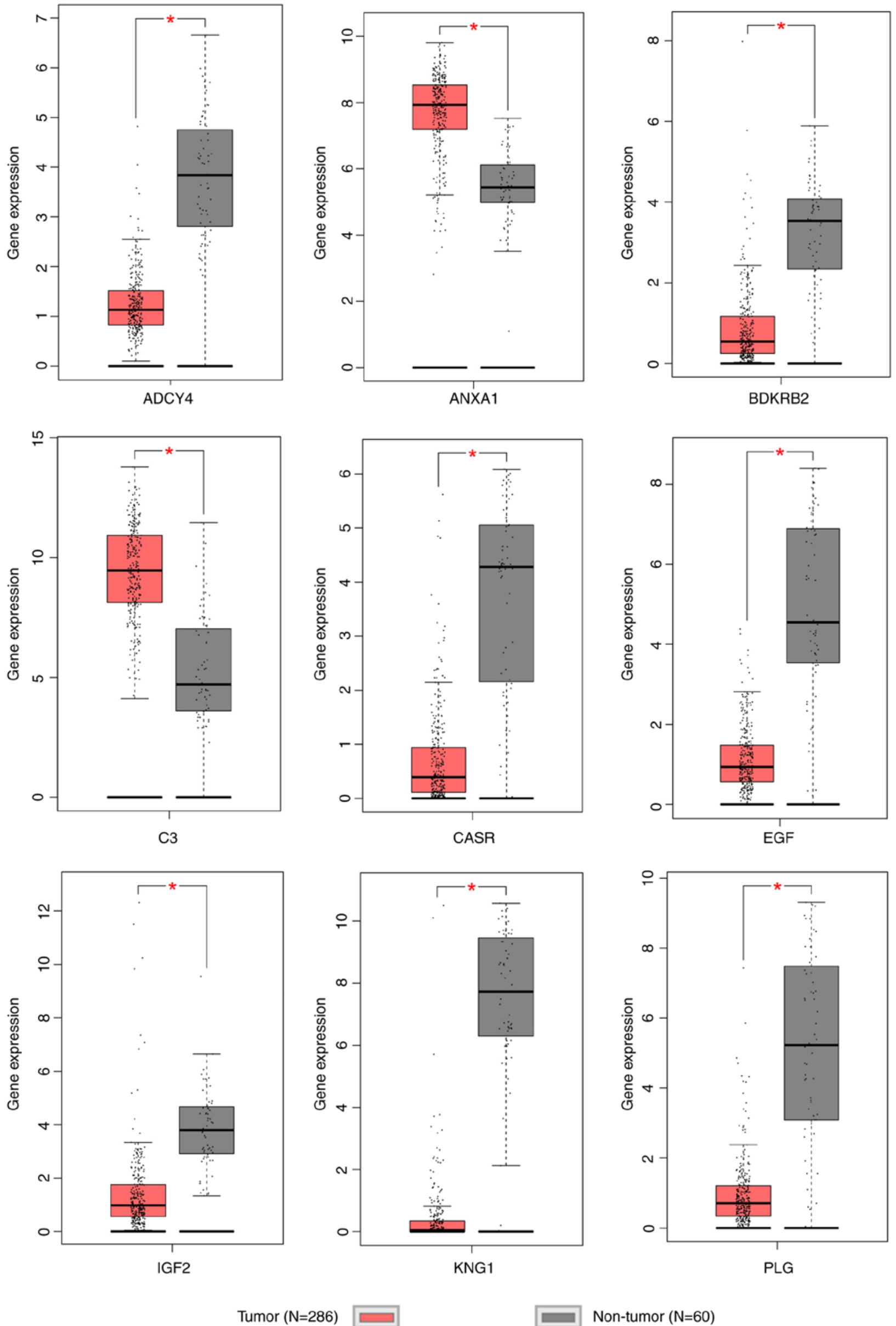

Figure 6. The expression difference of hub genes in the gene regulation network of papillary renal cell carcinoma. ${ }^{*} \mathrm{P}<0.05$. 

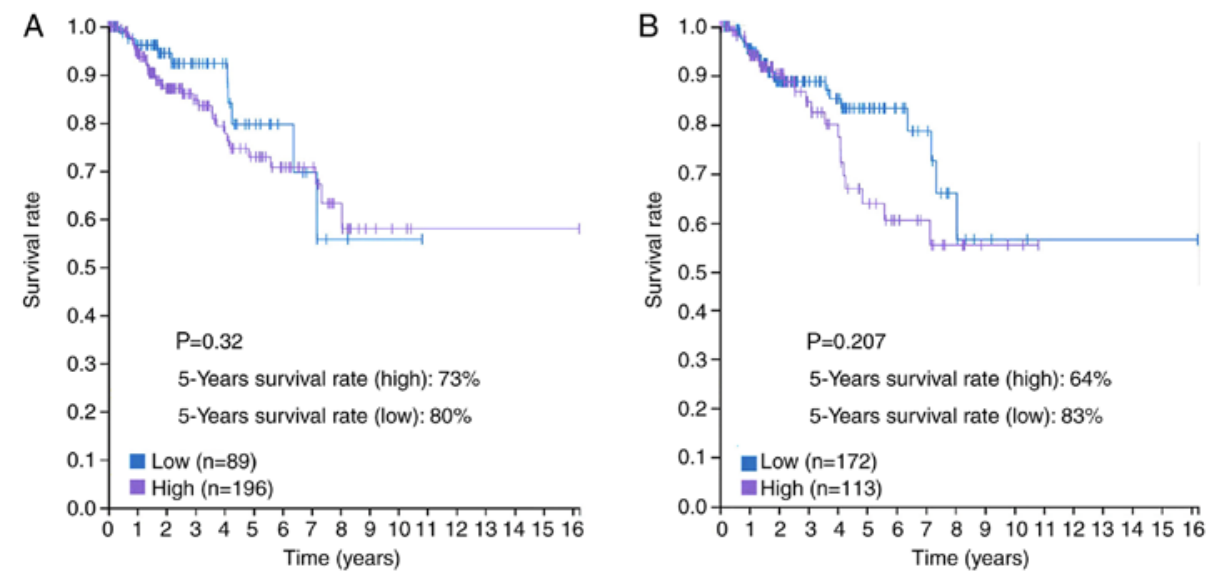

Figure 7. Prognostic value of (A) complement C3 and (B) annexin 1 in papillary renal cell carcinoma using The Cancer Genome Atlas data achieved from Protein Atlas (26).
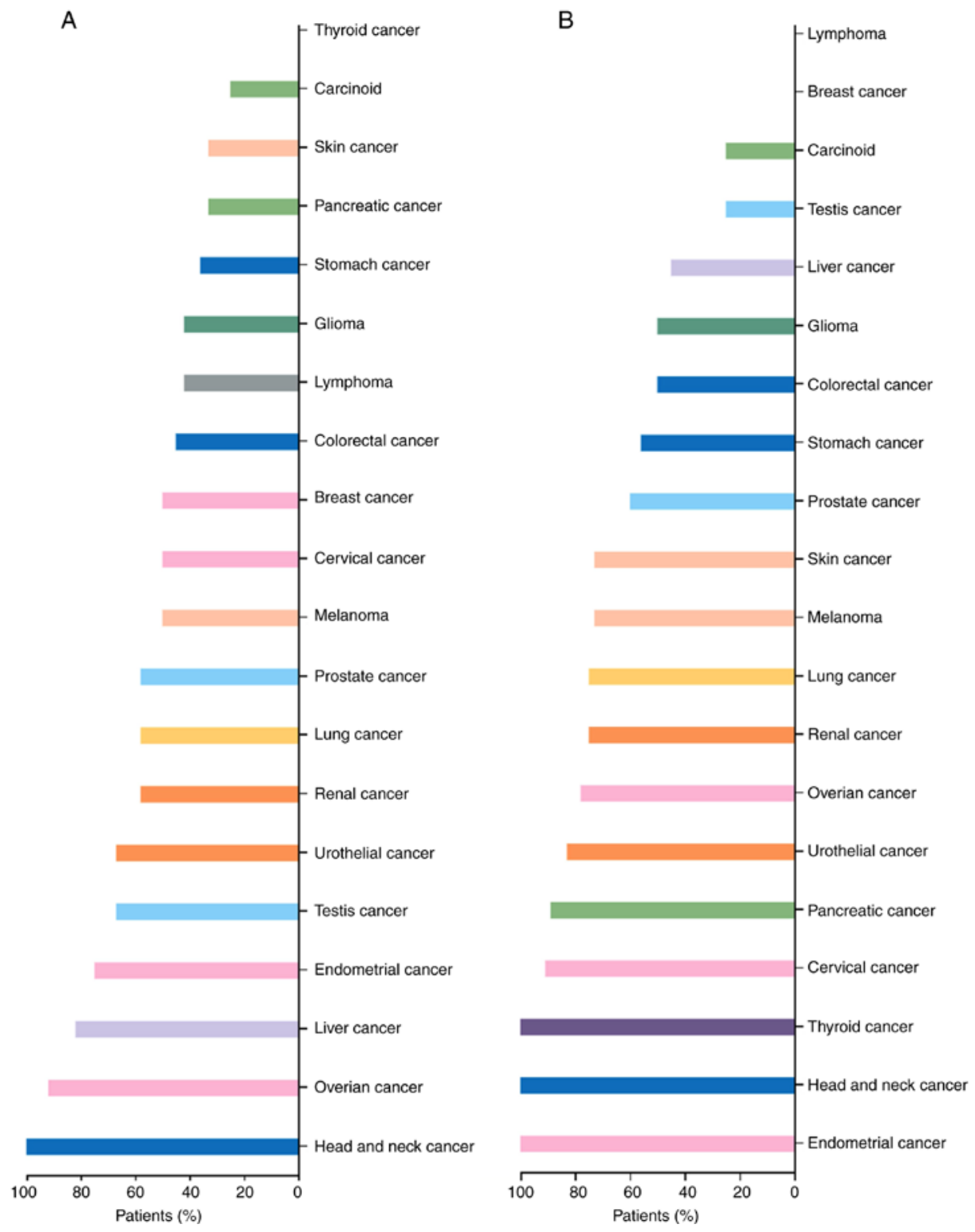

Figure 8. The validation of C3 and ANXA1 protein levels in various types of human cancers achieved from Protein Atlas (26). (A) Protein levels of C3 in human cancers. Distinct positivity was observed in stromal tumors. The majority of malignant cells displayed weak to moderate cytoplasmic immunoreactivity. Strong staining was found in ovarian, endometrial, testicular and renal cancers; (B) Protein levels of ANXA1 in human cancers. The majority of malignant cells displayed moderate to strong cytoplasmic and nuclear positivity. Hepatocellular carcinomas, basal cell carcinomas, breast cancers and malignant lymphomas were mainly weakly stained or negative. C3, complement C3; ANXA1, annexin 1. 
A

B
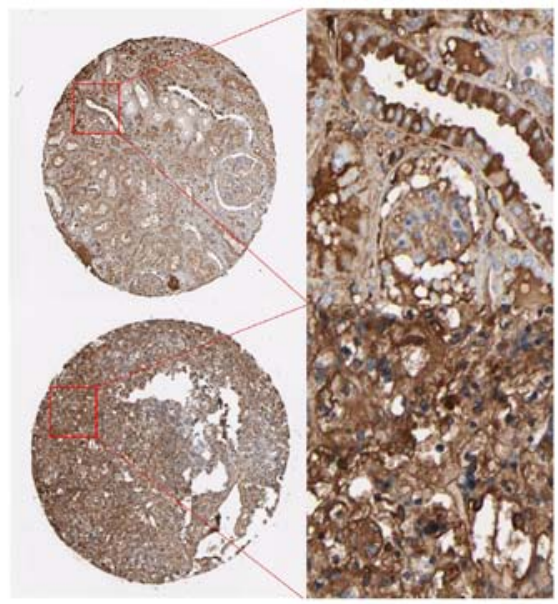

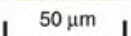

C

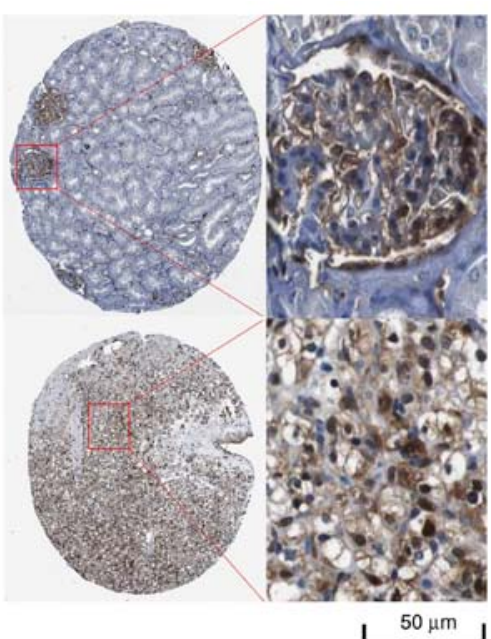

Figure 9. IHC results of C3 and ANXN1 protein levels in normal kidney and renal cancer from Protein Atlas (26). (A) IHC result of C3 protein in normal kidney. Staining. medium; intensity, moderate; quantity, 75-25\%; location, cytoplasmic/membranous. (B) IHC result of C3 protein in renal cancer. Staining, high; intensity, strong; quantity, 75-25\%; location, cytoplasmic/membranous. (C) IHC result of ANXA1 protein in normal kidney tissue. Staining, high; intensity, strong; quantity, 75-25\%; location, cytoplasmic/membranous/nuclear. (D) IHC result of ANXA1 protein in renal cancer. Staining, high; intensity, strong; quantity, 75-25\%; location, cytoplasmic/membranous/nuclear. IHC, immunohistochemical; C3, complement C3; ANXA1, annexin 1.

A

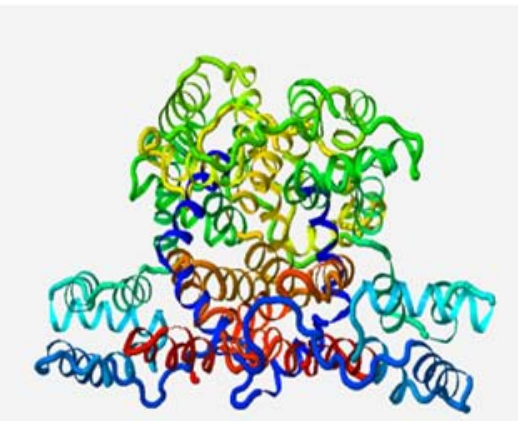

B
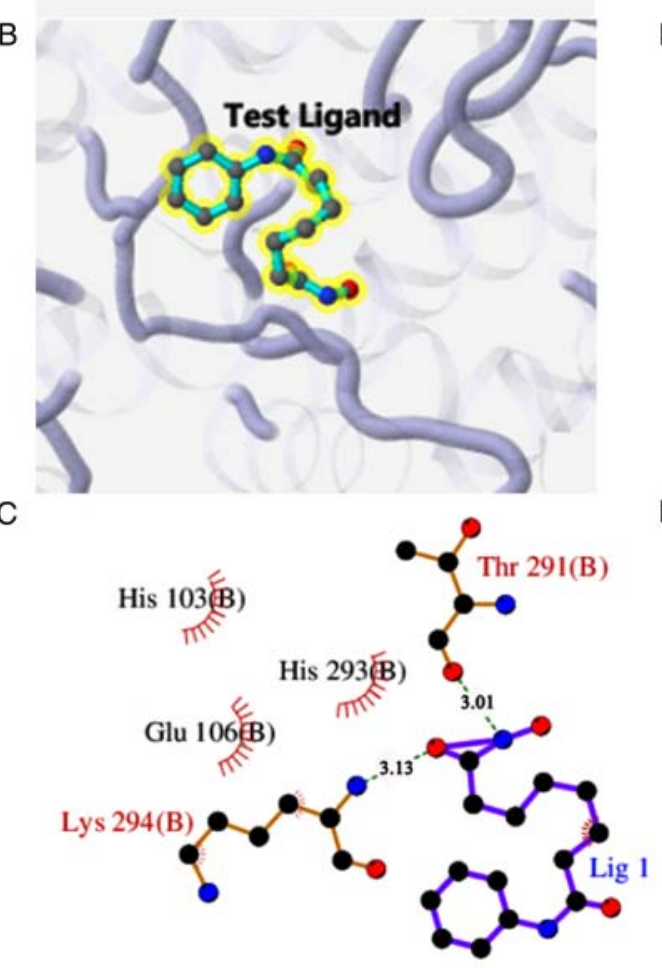

D

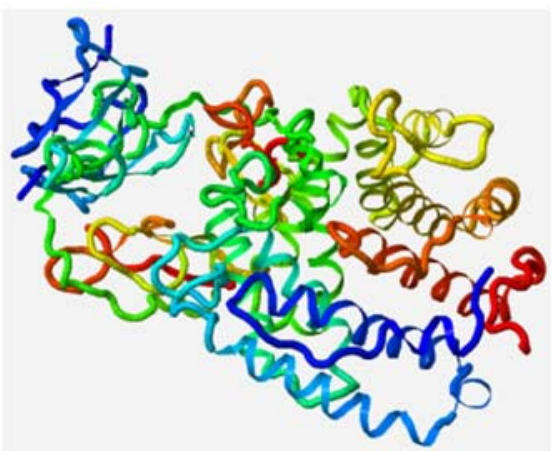

$\mathrm{E}$

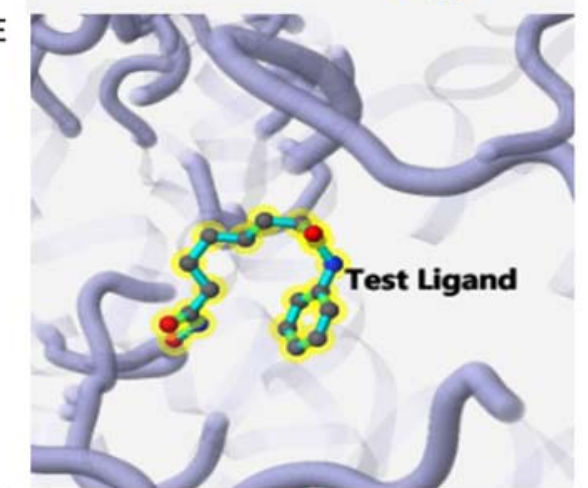

$\mathrm{F}$

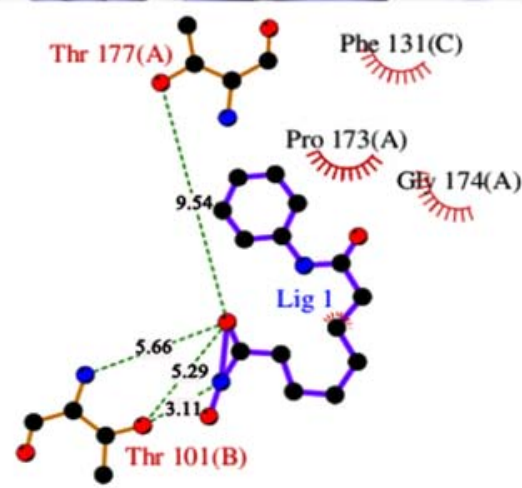

Figure 10. Molecular docking test for vorinostat and ANXA1 protein (PDB: 1HM6), and C3 protein (PDB: 1GHQ). (A) Protein structure of ANXN1. Drug-protein interaction between vorinostat and ANXA1 protein in (B) 3D and (C) 2D. (D) Protein structure of C3. Drug-protein interaction between vorinostat and $\mathrm{C} 3$ protein in (E) 3D and (F) 2D. C3, complement C3; ANXA1, annexin 1. 
Table IV. Computationally-predicted drugs that may improve the reversal effects of gene expression changes when combined with vorinostat in Drug Pair Seeker.

\begin{tabular}{|c|c|c|c|c|c|c|c|}
\hline Drug 1 & Drug 2 & $\begin{array}{c}\text { Total } \\
\text { coverage }\end{array}$ & $\begin{array}{c}\text { Total } \\
\text { conflicts }\end{array}$ & $\begin{array}{c}\text { Drug } 1 \\
\text { coverage }\end{array}$ & $\begin{array}{c}\text { Drug } 1 \\
\text { conflicts }\end{array}$ & $\begin{array}{c}\text { Drug } 2 \\
\text { coverage }\end{array}$ & $\begin{array}{c}\text { Drug } 2 \\
\text { conflicts }\end{array}$ \\
\hline Vorinostat-4444 & Propofol-3048 & 61 & 20 & 21 & 8 & 42 & 12 \\
\hline Vorinostat-4444 & Sulfamonomethoxine-2742 & 59 & 18 & 21 & 8 & 38 & 10 \\
\hline Vorinostat-4444 & Methazolamide-2733 & 58 & 18 & 21 & 8 & 38 & 10 \\
\hline Vorinostat-4444 & Phthalylsulfathiazole-5249 & 56 & 16 & 21 & 8 & 35 & 8 \\
\hline Vorinostat-4444 & Lobeline- 1770 & 49 & 11 & 21 & 8 & 35 & 8 \\
\hline Vorinostat-4444 & Parbendazole-3881 & 59 & 20 & 21 & 8 & 41 & 12 \\
\hline Vorinostat-4444 & Glipizide-6645 & 56 & 17 & 21 & 8 & 36 & 9 \\
\hline Vorinostat- 1220 & Propofol-3048 & 57 & 19 & 16 & 7 & 42 & 17 \\
\hline Vorinostat-4444 & Azacitidine-3348 & 57 & 19 & 21 & 8 & 38 & 13 \\
\hline Vorinostat-4444 & Rimexolone-5092 & 59 & 21 & 21 & 8 & 40 & 14 \\
\hline
\end{tabular}

Coverage refers to the number of favorable targets that the drug affects, meaning the gene expression level that the drug would reverse. Conflict refers to the number of genes the drug is potentially changing in an unfavorable outcome.

with vorinostat in PRCC treatment. Via this method, a total of 10 drugs (including propofol and sulfamonomethoxine) were revealed to have latent synergistic effects when combined with vorinostat (Table IV).

\section{Discussion}

In the present study, the DEGs of PRCC were identified using TCGA data and a bioinformatics analysis including GO analysis and KEGG pathway was performed to investigate the underlying mechanisms of PRCC. The identified DEGs were also used to search for potential drugs using the Cmap dataset for the treatment of PRCC. Subsequently, the potential application of query drugs in PRCC was further explored with the drug pathway network, drug-target network and a molecular docking test.

According to the GEPIA tool, 627 genes in total were considered as DEGs in PRCC, among which 161 were upregulated genes and 466 were downregulated genes. The GO functional annotation was performed based on these 627 DEGs by MetaScape and it demonstrated that these DEGs were mainly associated with anion transport, ion homeostasis, kidney development, and anion transmembrane transporter activity, which is consistent with other findings that suggest that ion transport has an essential role in tumor development and metastasis by altering substantially normal biological processes (33). The results from the KEGG enrichment pathway, as determined by WebGestalt, also reveal how these DEGS function in PRCC. Among the top 20 metabolic pathways, several significant pathways, such as complement and coagulation cascades and cell adhesion molecules (CAMs), are associated with human tumors $(34,35)$. Cancer migration originates from the disruption of cell adhesion interaction between cancer and normal cells/matrix, followed by an increased cell adhesion activity that interacts with other tissue. Therefore, CAMs is deemed to be a crucial pathway in the development and metastasis of human cancers (36-39). As previously reported, CAMs greatly contributes to migration and invasion in lung cancer, gastric cancer, and bladder cancer (40-42). In a previous study by Zimpfer et al (43), the overexpression of CAMs was located in 126 of 155 patients with PRCC and is clearly associated with higher grade and worse prognosis in PRCC patients. However, the majority of previous studies focused on the investigation of CCRCC, and to date there have been no published studies that investigate how CAMs pathway functions in PRCC based on the molecular mechanism. Therefore, more experiments are required to determine the importance of CAMs in PRCC, which may serve as an ignored therapeutic target in PRCC chemotherapy (44).

To identify more potential drugs for PRCC treatment, 60 candidate drugs were obtained from the prediction of the Cmap dataset depending on DEGs of PRCC. Among the top 10 drugs, vorinostat was particularly interesting and it is considered to be the most promising drug in PRCC treatment for detailed discussion.

Vorinostat, a histone deacetylase (HDAC) suppressor, has been widely applied for therapy in progressive cutaneous T-cell lymphoma via blocking cell cycle and/or inducing cell apoptosis that results from the accumulation of acetylated histone (29-32). In biology, DNA is wrapped around histones and its expression relies on the regulation of acetyltransferases and deacetylases (45). HDACs are a group of enzymes in eukaryotic nuclei that help histone deacetylation, and accordingly allow histones to assemble and transform DNA into bioactive units (46). It was reported that HDACs (HDAC1 and HDAC2) are required for cell growth and survival in RCC tumors (47). The inhibition of HDACs may reverse resistance to angiogenesis inhibitors and enhance oncologic chemotherapy responses in advanced RCC (48). A growing volume of evidence has suggested the incorporation of HDACs in the development of renal tumors, illustrating its decrease or suppression as a prospective therapeutic method to restrain renal tumors $(49,50)$. Recent studies suggest that vorinostat possesses antitumor activity against soft tissue sarcomas, 
gastric and lung cancer, and even RCC (51-54). In addition, the anti-virus effect of vorinostat in patients with HIV is also reported (55-57). For the safety of vorinostat in clinical application, a clinical trial published in 2017 suggested that the combination of bevacizumab and vorinostat is relatively safe and tolerated in patients with CCRCC (58). Chemotherapy effects of vorinostat for patients with PRCC, however, are still not confirmed by clinical trials. Regarding other drugs, such as naftifine, amiodarone and valproic acid, the antitumor effect of these drugs in human cancers has also been reported in recent years (59-66).

In the present study, the results of drug prediction in Cmap suggest that vorinostat had a relatively low connectivity score, which indicates a high inverse correlation between vorinostat and DEGs of PRCC. From the prediction of drug targets, it was observed that vorinostat is directly targeted to TP53, and there have been a number of published studies, that argue that the mutation of TP53 greatly contributes to the tumorigenesis and development of RCC (67-69). The current study also observed that vorinostat exerts a significant influence in regulating the p53 and MAPK signaling pathway. Previous studies have indicated that both $\mathrm{p} 53$ and MAPK signaling pathway are clearly associated with various cellular functions, including apoptosis, cell growth, migration and induction of aging, and serve as key pathways for tumorigenesis and progression in kidney cancers (68,70-74). Therefore, vorinostat may possess an antitumor activity by inhibiting p53 and MAPK signaling pathway.

For a better investigation of the specific molecular mechanism and potential application of vorinostat in anti-PRCC activity, a PPI network was constructed to search for hub genes in the gene regulation network of PRCC. From the PPI network, 9 genes are considered to be hub genes in the DEGs of PRCC, in which 2 genes, $\mathrm{C} 3$ and ANXN1, are significantly upregulated. Notably, there was no statistical difference in survival curves for C3 and ANXN1 to support their role in the prognosis of PRCC patients. However, these 2 genes are considered as oncologic therapeutic targets in the PRCC treatment as their key roles of hub genes in the regulation network of DEGs, therefore, the suppression of these 2 genes may interfere with a series of interactions between the DEGs, thereby inhibiting the development and progression of PRCC and then help to treat patients with PRCC. A target drug performs its effects on cancer cells via interaction with the specific proteins encoded by oncogenic or key genes, therefore, molecular docking tests were performed to investigate the drug-protein interactions between vorinostat and these two proteins ( $\mathrm{C} 3$ and ANXN1). The results provided by systemDock precisely simulate their interaction patterns and illustrate how vorinostat acts on $\mathrm{C} 3$ and ANXN1 proteins in the human body. Usually, the binding ability of small molecules and proteins are evaluated by docking scores. Surprisingly, the results as observed from docking tests demonstrate that vorinostat can recognize and interact with both $\mathrm{C} 3$ and ANXN1 proteins (docking tests $>4$ ), which suggests that vorinostat has a considerably prospective performance in PRCC treatment by suppressing the regulation network of DEGs through inhibiting C3 and ANXN1 proteins. In the future, more experimental evidence and long-term clinical trials are required to validate the effects of vorinostat in PRCC treatment.
Some limitations in the present study remain to be answered. First, the hub genes verified from the PPI network should be further validated in vitro to observe their specific role in PRCC. Second, the effect of potential drugs from Cmap prediction for PRCC treatment should also be further investigated using experimental evidence.

In summary, disregarding the above limitations, 627 DEGs have been identified in PRCC based on TCGA data in the current study, and the underlying mechanism of PRCC has been further investigated by GO and KEGG pathway analysis. Furthermore, to the best of our knowledge, the present study was the first to predict 60 candidate drugs for PRCC treatment based on DEGs by integrating Cmap dataset, in which vorinostat was considered to be the most prospective drug and exhibited significant anti-PRCC activity by inhibiting the regulation network of DEGs by targeting C3 and ANXA1.

\section{Acknowledgements}

Not applicable.

\section{Funding}

The present study was supported by the Fund of Promoting Project of Basic Capacity for Young and Middle-aged University Teachers in Guangxi (grant no. KY2016LX034) and the Medical Excellence Award Funded by the Creative Research Development Grant from the First Affiliated Hospital of Guangxi Medical University.

\section{Availability of data and materials}

The datasets used and/or analyzed during the current study are available from the corresponding author on reasonable request.

\section{Authors' contributions}

GC, HBY, and SHL involved in the conception and design of the study, as well as designed the figures and tables. JSP, PL, XDW, and ZKL contributed to the statistical analysis, as well as wrote and corrected the manuscript. All authors read and approved the final manuscript.

\section{Ethics approval and consent to participate}

Not applicable.

\section{Patient consent for publication}

Not applicable.

\section{Competing interests}

The authors declare that they have no competing interests.

\section{References}

1. Wu X, Liu D, Gao X, Xie F, Tao D, Xiao X, Wang L, Jiang G and Zeng F: Inhibition of BRD4 suppresses cell proliferation and induces apoptosis in renal cell carcinoma. Cell Physiol Biochem 41: 1947-1956, 2017. 
2. Wang C, Cai L, Liu J, Wang G, Li H, Wang X, Xu W, Ren M, Feng L, Liu P, et al: MicroRNA-30a-5p inhibits the growth of renal cell carcinoma by modulating GRP78 expression. Cell Physiol Biochem 43: 2405-2419, 2017.

3. Wang J, Li M, Wang Y and Liu X: Integrating subpathway analysis to identify candidate agents for hepatocellular carcinoma. Onco Targets Ther 9: 1221-1230, 2016.

4. Siegel RL, Miller KD and Jemal A: Cancer statistics, 2018. CA Cancer J Clin 68: 7-30, 2018.

5. Steffens S, Janssen M, Roos FC, Becker F, Schumacher S, Seidel C, Wegener G, Thüroff JW, Hofmann R, Stöckle M, et al: Incidence and long-term prognosis of papillary compared to clear cell renal cell carcinoma-a multicentre study. Eur J Cancer 48 : 2347-2352, 2012

6. Lan H, Zeng J, Chen G and Huang H: Survival prediction of kidney renal papillary cell carcinoma by comprehensive LncRNA characterization. Oncotarget 8: 110811-110829, 2017.

7. Sourbier C, Liao PJ, Ricketts CJ, Wei D, Yang Y, Baranes SM, Gibbs BK, Ohanjanian L, Spencer Krane L, Scroggins BT, et al: Targeting loss of the Hippo signaling pathway in NF2-deficient papillary kidney cancers. Oncotarget 9: 10723-10733, 2018.

8. Chakraborty S, Tarantolo SR, Batra SK and Hauke RJ: Incidence and prognostic significance of second primary cancers in renal cell carcinoma. Am J Clin Oncol 36: 132-142, 2013.

9. De P, Otterstatter MC, Semenciw R, Ellison LF, Marrett LD and Dryer D: Trends in incidence, mortality, and survival for kidney cancer in Canada, 1986-2007. Cancer Causes Control 25 1271-1281, 2014

10. Ke X, Zeng X, Wei X, Shen Y, Gan J, Tang H and Hu Z: MiR-514a-3p inhibits cell proliferation and epithelial-mesenchymal transition by targeting EGFR in clear cell renal cell carcinoma. Am J Transl Res 9: 5332-5346, 2017.

11. Akhtar M, Al-Bozom IA and Al Hussain T: Papillary renal cell carcinoma (PRCC): An update. Adv Anat Pathol: Nov 30, 2018 (Epub ahead of print). doi: 10.1097/PAP.0000000000000220.

12. Courthod G, Tucci M, Di Maio M and Scagliotti GV: Papillary renal cell carcinoma: A review of the current therapeutic landscape. Crit Rev Oncol Hematol 96: 100-112, 2015.

13. Sleire L, Førde HE, Netland IA, Leiss L, Skeie BS and Enger PØ Drug repurposing in cancer. Pharmacol Res 124: 74-91, 2017.

14. Tang Z, Li C, Kang B, Gao G, Li C and Zhang Z: GEPIA: A web server for cancer and normal gene expression profiling and interactive analyses. Nucleic Acids Res 45: W98-W102, 2017

15. Zhang Y, Huang JC, Cai KT, Yu XB, Chen YR, Pan WY, He ZL, Lv J, Feng ZB and Chen G: Long noncoding RNA HOTTIP promotes hepatocellular carcinoma tumorigenesis and development: A comprehensive investigation based on bioinformatics, qRTPCR and metaanalysis of 393 cases. Int J Oncol 51: 1705-1721, 2017.

16. He Z, Tang F, Lu Z, Huang Y, Lei H, Li Z and Zeng G: Analysis of differentially expressed genes, clinical value and biological pathways in prostate cancer. Am J Transl Res 10: 1444-1456, 2018.

17. Yang X, Zhu S, Li L, Zhang L, Xian S, Wang Y and Cheng Y: Identification of differentially expressed genes and signaling pathways in ovarian cancer by integrated bioinformatics analysis. Onco Targets Ther 11: 1457-1474, 2018.

18. Tripathi S, Pohl MO, Zhou Y, Rodriguez-Frandsen A, Wang G, Stein DA, Moulton HM, DeJesus P, Che J, Mulder LC, et al: Meta- and orthogonal integration of influenza 'OMICs' data defines a role for UBR4 in virus budding. Cell Host Microbe 18 : 723-735, 2015

19. Wang J, Vasaikar S, Shi Z, Greer M and Zhang B: WebGestalt 2017: A more comprehensive, powerful, flexible and interactive gene set enrichment analysis toolkit. Nucleic Acids Res 45: W130-W137, 2017.

20. Lamb J, Crawford ED, Peck D, Modell JW, Blat IC, Wrobel MJ, Lerner J, Brunet JP, Subramanian A, Ross KN, et al: The Connectivity Map: Using gene-expression signatures to connect small molecules, genes, and disease. Science 313: 1929-1935, 2006.

21. Qu XA and Rajpal DK: Applications of Connectivity Map in drug discovery and development. Drug Discov Today 17: 1289-1298, 2012

22. Zhong Y, Chen EY, Liu R, Chuang PY, Mallipattu SK, Tan CM, Clark NR, Deng Y, Klotman PE, Ma'ayan A, et al: Renoprotective effect of combined inhibition of angiotensin-converting enzyme and histone deacetylase. J Am Soc Nephrol 24: 801-811, 2013.
23. Li C, Li X, Miao Y, Wang Q, Jiang W, Xu C, Li J, Han J, Zhang F, Gong B, et al: SubpathwayMiner: A software package for flexible identification of pathways. Nucleic Acids Res 37: e131, 2009.

24. Szklarczyk D, Santos A, von Mering C, Jensen LJ, Bork P and Kuhn M: STITCH 5: Augmenting protein-chemical interaction networks with tissue and affinity data. Nucleic Acids Res 44: D380-D384, 2016.

25. Szklarczyk D, Morris JH, Cook H, Kuhn M, Wyder S, Simonovic M, Santos A, Doncheva NT, Roth A, Bork P, et al: The STRING database in 2017: Quality-controlled protein-protein association networks, made broadly accessible. Nucleic Acids Res 45: D362-D368, 2017

26. Scardoni G, Tosadori G, Faizan M, Spoto F, Fabbri F and Laudanna C: Biological network analysis with CentiScaPe: Centralities and experimental dataset integration. F1000Res 3: 139, 2014

27. Interactive human protein atlas launches. Cancer Discov 5: 339 , 2015.

28. Hsin KY, Matsuoka Y, Asai Y, Kamiyoshi K, Watanabe T, Kawaoka Y and Kitano H: systemsDock: A web server for network pharmacology-based prediction and analysis. Nucleic Acids Res 44: W507-W513, 2016.

29. Han L, Wang T, Wu J, Yin X, Fang $\mathrm{H}$ and Zhang $\mathrm{N}$ : A facile route to form self-carried redox-responsive vorinostat nanodrug for effective solid tumor therapy. Int J Nanomedicine 11: 6003-6022, 2016.

30. Fernández-Rodríguez C, Salar A, Navarro A, Gimeno E, Pairet S, Camacho L, Ferraro M, Serrano S, Besses C, Bellosillo B, et al: Anti-tumor activity of the combination of bendamustine with vorinostat in diffuse large B-cell lymphoma cells. Leuk Lymphoma 57: 692-699, 2016.

31. Duvic $M$ and Dimopoulos $M$ : The safety profile of vorinostat (suberoylanilide hydroxamic acid) in hematologic malignancies: A review of clinical studies. Cancer Treat Rev 43: 58-66, 2016.

32. Zinzani PL, Bonthapally V, Huebner D, Lutes R, Chi A and Pileri S: Panoptic clinical review of the current and future treatment of relapsed/refractory T-cell lymphomas: Cutaneous T-cell lymphomas. Crit Rev Oncol Hematol 99: 228-240, 2016.

33. Djamgoz MB, Coombes RC and Schwab A: Ion transport and cancer: From initiation to metastasis. Philos Trans R Soc Lond B Biol Sci 369: 20130092, 2014.

34. Guglietta S and Rescigno M: Hypercoagulation and complement: Connected players in tumor development and metastases. Semin Immunol 28: 578-586, 2016.

35. Samatov TR, Wicklein D and Tonevitsky AG: L1CAM: Cell adhesion and more. Prog Histochem Cytochem 51: 25-32, 2016.

36. Kourtidis A and Anastasiadis PZ: Bringing together cell-to-cell adhesion and miRNA biology in cancer research. Future Oncol 12: 1211-1214, 2016.

37. Brower V: Adhesion molecules, stem cells, and the microenvironment in acute myeloid leukemia. J Natl Cancer Inst 108: djw113, 2016.

38. Turaga SM and Lathia JD: Adhering towards tumorigenicity: Altered adhesion mechanisms in glioblastoma cancer stem cells. CNS Oncol 5: 251-259, 2016.

39. Hamidi $\mathrm{H}$ and Ivaska J: Every step of the way: Integrins in cancer progression and metastasis. Nat Rev Cancer 18: 533-548, 2018.

40. Tang Q, Zhang H, Kong M, Mao X and Cao X: Hub genes and key pathways of non-small lung cancer identified using bioinformatics. Oncol Lett 16: 2344-2354, 2018.

41. Zhang Y, Zhang J, Shen Q, Yin W, Huang H, Liu Y and Ni Q: High expression of Nectin-4 is associated with unfavorable prognosis in gastric cancer. Oncol Lett 15: 8789-8795, 2018.

42. Chang HY, Chang HM, Wu TJ, Chaing CY, Tzai TS, Cheng HL, Raghavaraju G, Chow NH and Liu HS: The role of Lutheran/basal cell adhesion molecule in human bladder carcinogenesis. J Biomed Sci 24: 61, 2017.

43. Zimpfer A, Maruschke M, Rehn S, Kundt G, Litzenberger A, Dammert F, Zettl H, Stephan C, Hakenberg OW and Erbersdobler A: Prognostic and diagnostic implications of epithelial cell adhesion/activating molecule (EpCAM) expression in renal tumours: A retrospective clinicopathological study of 948 cases using tissue microarrays. BJU Int 114: 296-302, 2014.

44. Alimbretov D, Askarova S, Umbayev B, Davis T and Kipling D: Pharmacological targeting of cell cycle, apoptotic and cell adhesion signaling pathways implicated in chemoresistance of cancer cells. Int J Mol Sci 19: E1690, 2018. 
45. Shechter D, Dormann HL, Allis CD and Hake SB: Extraction, purification and analysis of histones. Nat Protoc 2: 1445-1457, 2007.

46. Valenzuela-Fernández A, Cabrero JR, Serrador JM and Sánchez-Madrid F: HDAC6: A key regulator of cytoskeleton, cell migration and cell-cell interactions. Trends Cell Biol 18: 291-297, 2008.

47. Kiweler N, Brill B, Wirth M, Breuksch I, Laguna T, Dietrich C, Strand S, Schneider G, Groner B, Butter F, et al: The histone deacetylases HDAC1 and HDAC2 are required for the growth and survival of renal carcinoma cells. Arch Toxicol 92: 2227-2243, 2018.

48. Aggarwal R, Thomas S, Pawlowska N, Bartelink I, Grabowsky J, Jahan T, Cripps A, Harb A, Leng J, Reinert A, et al: Inhibiting histone deacetylase as a means to reverse resistance to angiogenesis inhibitors: Phase I study of abexinostat plus pazopanib in advanced solid tumor malignancies. J Clin Oncol 35: 1231-1239, 2017.

49. Chun P: Therapeutic effects of histone deacetylase inhibitors on kidney disease. Arch Pharm Res 41: 162-183, 2018.

50. Dokmanovic M, Clarke C and Marks PA: Histone deacetylase inhibitors: Overview and perspectives. Mol Cancer Res 5: 981-989, 2007.

51. Schmitt T, Mayer-Steinacker R, Mayer F, Grünwald V, Schütte J, Hartmann JT, Kasper B, Hüsing J, Hajda J, Ottawa G, et al: Vorinostat in refractory soft tissue sarcomas-Results of a multi-centre phase II trial of the German Soft Tissue Sarcoma and Bone Tumour Working Group (AIO). Eur J Cancer 64: 74-82, 2016

52. Pan CH, Chang YF, Lee MS, Wen BC, Ko JC, Liang SK and Liang MC: Vorinostat enhances the cisplatin-mediated anticancer effects in small cell lung cancer cells. BMC Cancer 16: $857,2016$.

53. Yoo C, Ryu MH, Na YS, Ryoo BY, Lee CW and Kang YK: Vorinostat in combination with capecitabine plus cisplatin as a first-line chemotherapy for patients with metastatic or unresectable gastric cancer: Phase II study and biomarker analysis. Br J Cancer 114: 1185-1190, 2016.

54. Li H, Wang X, Zhang C, Cheng Y, Yu M, Zhao K, Ge W, Cai A, Zhang Y, Han F, et al: HDAC1-induced epigenetic silencing of ASPP 2 promotes cell motility, tumour growth and drug resistance in renal cell carcinoma. Cancer Lett 432: 121-131, 2018.

55. Archin NM, Liberty AL, Kashuba AD, Choudhary SK, Kuruc JD, Crooks AM, Parker DC, Anderson EM, Kearney MF, Strain MC, et al: Administration of vorinostat disrupts HIV-1 latency in patients on antiretroviral therapy. Nature 487: 482-485, 2012.

56. Sung JA, Sholtis K, Kirchherr J, Kuruc JD, Gay CL, Nordstrom JL, Bollard CM, Archin NM and Margolis DM: Vorinostat renders the replication-competent latent reservoir of human immunodeficiency virus (HIV) vulnerable to clearance by CD8 T cells. EBioMedicine 23: 52-58, 2017. J Clin Invest 127: 3126-3135, 2017.

57. Mota TM, Rasmussen TA, Rhodes A, Tennakoon S, Dantanarayana A, Wightman F, Hagenauer M, Roney J, Spelman T, Purcell DFJ, et al: No adverse safety or virological changes 2 years following vorinostat in HIV-infected individuals on antiretroviral therapy. AIDS 31: 1137-1141, 2017.

58. Pili R, Liu G, Chintala S, Verheul H, Rehman S, Attwood K, Lodge MA, Wahl R, Martin JI, Miles KM, et al: Combination of the histone deacetylase inhibitor vorinostat with bevacizumab in patients with clear-cell renal cell carcinoma: A multicentre, single-arm phase I/II clinical trial. Br J Cancer 116: 874-883, 2017.

59. Schmeel LC, Schmeel FC, Blaum-Feder S and Schmidt-Wolf IG: In vitro efficacy of naftifine against lymphoma and multiple myeloma. Anticancer Res 35: 5921-5926, 2015.

60. Bognar Z, Fekete K, Antus C, Hocsak E, Bognar R, Tapodi A, Boronkai A, Farkas N, Gallyas F Jr, Sumegi B, et al: Desethylamiodarone-A metabolite of amiodarone-Induces apoptosis on T24 human bladder cancer cells via multiple pathways. PLoS One 12: e0189470, 2017.
61. Chang YL, Liu ST, Wang YW, Lin WS and Huang SM: Amiodarone promotes cancer cell death through elevated truncated SRSF3 and downregulation of miR-224. Oncotarget 9: 13390-13406, 2018.

62. Felix F and Fontenele J: Valproic acid may be tested in patients with $H 3 F 3 A$-mutated high-grade gliomas. J Clin Oncol 34: 3104-3105, 2016

63. Cha HY, Lee BS, Chang JW, Park JK, Han JH, Kim YS, Shin YS, Byeon HK and Kim CH: Downregulation of Nrf2 by the combination of TRAIL and Valproic acid induces apoptotic cell death of TRAIL-resistant papillary thyroid cancer cells via suppression of Bcl-xL. Cancer Lett 372: 65-74, 2016.

64. Xi W, Chen X, Sun J, Wang W, Huo Y, Zheng G, Wu J, Li Y, Yang $A$ and Wang T: Combined treatment with valproic acid and 5-Aza-2'-deoxycytidine synergistically inhibits human clear cell renal cell carcinoma growth and migration. Med Sci Monit 24: 1034-1043, 2018.

65. Wei M, Mao S, Lu G, Li L, Lan X, Huang Z, Chen Y, Zhao M, Zhao Y and Xia Q: Valproic acid sensitizes metformin-resistant human renal cell carcinoma cells by upregulating $\mathrm{H} 3$ acetylation and EMT reversal. BMC Cancer 18: 434, 2018.

66. Mao S, Lu G, Lan X, Yuan C, Jiang W, Chen Y, Jin X and Xia Q: Valproic acid inhibits epithelialmesenchymal transition in renal cell carcinoma by decreasing SMAD4 expression. Mol Med Rep 16: 6190-6199, 2017.

67. Tajima S, Waki M, Doi W, Hayashi K, Takenaka S, Fukaya Y and Kimura R: Acquired cystic disease-associated renal cell carcinoma with a focal sarcomatoid component: Report of a case showing more pronounced polysomy of chromosomes 3 and 16 in the sarcomatoid component. Pathol Int 65: 89-94, 2015.

68. Hayes SA, Pandiri AR, Ton TV, Hong HH, Clayton NP, Shockley KR, Peddada SD, Gerrish K, Wyde M, Sills RC, et al: Renal cell carcinomas in vinylidene chloride-exposed male B6C3F1 mice are characterized by oxidative stress and TP53 pathway dysregulation. Toxicol Pathol 44: 71-87, 2016.

69. Malouf GG, Ali SM, Wang K, Balasubramanian S, Ross JS, Miller VA, Stephens PJ, Khayat D, Pal SK, Su X, et al: Genomic characterization of renal cell carcinoma with sarcomatoid dedifferentiation pinpoints recurrent genomic alterations. Eur Urol 70: 348-357, 2016.

70. Casuscelli J, Weinhold N, Gundem G, Wang L, Zabor EC, Drill E, Wang PI, Nanjangud GJ, Redzematovic A, Nargund AM, et al: Genomic landscape and evolution of metastatic chromophobe renal cell carcinoma. JCI insight 2: 92688, 2017.

71. Gao Y, Li J, Qiao N, Meng Q, Zhang M, Wang X, Jia J, Yang S, Qu C, Li W, et al: Adrenomedullin blockade suppresses sunitinib-resistant renal cell carcinoma growth by targeting the ERK/MAPK pathway. Oncotarget 7: 63374-63387, 2016.

72. Miyazaki A, Miyake H and Fujisawa M: Molecular mechanism mediating cytotoxic activity of axitinib in sunitinib-resistant human renal cell carcinoma cells. Clin Transl Oncol 18: 893-900, 2016.

73. Ye H, Wang WG, Cao J and Hu XC: SPARCL1 suppresses cell migration and invasion in renal cell carcinoma. Mol Med Rep 16: 7784-7790, 2017.

74. Ohnami S, Ohshima K, Nagashima T, Urakami K, Shimoda Y, Saito J, Naruoka A, Hatakeyama K, Mochizuki T, Serizawa M, et al: Comprehensive characterization of genes associated with the TP53 signal transduction pathway in various tumors. Mol Cell Biochem 431: 75-85, 2017.

This work is licensed under a Creative Commons Attribution-NonCommercial-NoDerivatives 4.0 International (CC BY-NC-ND 4.0) License. 